\title{
Efecto de tres enjuagues bucales en la degradación superficial de resinas compuestas: estudio in vitro
}

Paola Elisabeth Casanova Obando';

María Fernanda Taboada Alvear ${ }^{2}$;

David Sebastián Flores Cuvi;

Marisol Castilla;

Ana del Carmen Armas ${ }^{5}$

\section{Resumen}

Objetivo: Evaluar la degradación de resinas compuestas en cuanto a su masa y rugosidad, al ser sometidas al contacto con enjuagues bucales. Materiales y Métodos: 88 discos de resinas nanohíbridas Grandio (VOCO) y Filtek Z250 XT (3M), correspondieron 44 discos para cada resina, a su vez se dividieron en 3 subgrupos (n: 14) y 2 discos seleccionados aleatoriamente fueron considerados control. Fueron sumergidos en un mismo enjuague bucal, Listerine Zero, Coolmint, y Whitening por 21 minutos, seguido por un nuevo pesaje y evaluación de la rugosidad superficial, para luego ser colocados en contacto con saliva artificial por 12 horas, entrando nuevamente en contacto con el enjuague respectivo y ser sometidos a una nueva evaluación de peso y rugosidad. Cada uno de estos ciclos fue ejecutado hasta completar 1092 minutos a una temperatura ambiente de $37^{\circ} \mathrm{C}$. Resultados: las mediciones de rugosidad y peso analizados mediante ANOVA $(p<0,001)$, mostraron ausencia de cambios significativos en las resinas evaluadas luego de ser sometidas al desafío del contacto con los enjuagues, sin embargo, sí se encontró diferencia en relación al tiempo de contacto. Conclusiones: los enjuagues bucales pueden provocar efecto negativo sobre las resinas compuestas que se incrementan en relación al tiempo de contacto.

Palabra clave: Resinas compuestas, antisépticos bucales, propiedades de superficie, pérdida de masa.

\footnotetext{
1. Odontóloga, Estudiante posgrado Rehabilitación Oral Facultad de Odontología, Universidad Central del Ecuador, Quito, Ecuador.

2. Odontóloga, Estudiante posgrado Rehabilitación Oral Facultad de Odontología, Universidad Central del Ecuador, Quito, Ecuador.

3. Odontólogo, Estudiante posgrado Rehabilitación Oral Facultad de Odontología, Universidad Central del Ecuador, Quito, Ecuador.

4. MSc. PhD, Docente Facultad de Odontología, Universidad Peruana Cayetano Heredia, Lima, Perú.

5. MSc. PhD, Docente Facultad de Odontología, Universidad Central del Ecuador, Quito, Ecuador.
} 
Artigo Original

\section{Efeito de três águas bocais sobre degradação de superfície de resina composto: estudo in vitro}

\section{Resumo}

Objetivo: Avaliar a degradação das resinas compostas quanto a sua massa e rugosidade, quando em contato com colutórios bucais. Materiais e Métodos: 88 discos de resinas nanohíbridas Grandio (VOCO) e Z250 XT (3M), sendo 44 discos para cada resina, que foram divididas em 3 grupos (n: 14) e dois discos, escolhidos aleatoriamente, foram consideradas controle. As amostras foram imersas nos seguintes colutórios: Listerine zero, Coolmint e branqueamento durante 21 minutos, em seguida foi realizada uma nova pesagem e avaliação da rugosidade da superfície, e, por fim, foram colocados em contato com a saliva artificial du- rante 12 horas. Após esse periodo, as amostras foram submetida a uma reavaliação do massa e rugosidade. Cada um destes ciclos foi executado para completar 1.092 minutos a uma temperatura ambiente de $37^{\circ} \mathrm{C}$. Resultados: medições da rugosidade e analisados por ANOVA $(p<0,001)$ não revelou alterações significativas nas resinas avaliadas, no entanto, outras diferenças foram encontradas em relação ao tempo contato. Conclusões: Os colutórios podem causar efeito negativo sobre as resinas compostas conforme o aumento de tempo de uso.

Palavra-chave: resinas compostas, anti-sépticos orais, propriedades de superfície, perda de massa.

Original article

\section{Effect of three mouthrinses on surface degradation of resin composite: in vitro study}

\begin{abstract}
Aim: Evaluate the degradation of composite resins in terms of mass and roughness, when it is in contact with mouth rinses. Materials and Methods: 88 discs of nanohybrid resins Grandio (VOCO) and Filtek Z250 XT (3M), corresponded to 44 discs for each one, divided
\end{abstract}

into 3 subgroups (n: 14) and 2 discs that were randomly selected as control group. They were submerged in the same mouth rinse, Listerine Zero, Coolmint, and Whitening for 21 minutes, followed by a new weighing and evaluation of the surface roughness, then they were placed in contact with artificial saliva for 12 hours, and then placed again in contact with the respective mouth rinse and also be subjected to a 
new evaluation of weight and roughness. Each of these cycles was executed until 1092 minutes were completed at a temperature of $37^{\circ} \mathrm{C}$. Results: The roughness and weight measurements analyzed by ANOVA $(\mathrm{p}<0,001)$ showed no significant changes in the evaluated resins after the contact of mouth rinses, neverthe-

\section{Introducción}

En la actualidad, la estética dental ocupa un lugar predominante en la población y las diferentes técnicas restauradoras con los materiales resinosos brindan soluciones satisfactorias y de buena aceptación por parte de los pacientes, asociada a mejoras en su estructura y propiedades. ${ }^{1}$ Relacionadas con la incorporación de partículas de relleno en acuerdo a la forma y tamaño ${ }^{2}$ que han proporcionado un excelente pulido, adecuada superficie, retención de brillo, alta resistencia a la abrasión, mayor dureza, contracción de polimerización reducida y menor filtración marginal de forma proporcional a las mejoras ejecutadas. ${ }^{3,4}$

Pese a las bondades del material, factores como un exceso de sorción acuosa y solubilidad, son efectos negativos en sus propiedades, desencadenando una degradación hidrolítica., Ésta es el resultado de la ruptura de enlaces químicos en la resina o el ablandamiento a través de la acción plastificante del agua. ${ }^{6}$ Adicionalmente algunos de los componentes, tales como los monómeros sin reaccionar, se disuelven y se eliminan por lixiviación, dando como resultado una pérdida de peso produciendo una solubilidad y definiendo a la captación de agua que produce un aumento de peso. ${ }^{7}$

A su vez, la absorción se entiende como la capacidad de penetración de sustancias como saliva, less, there was significant in relation with the time of contact. Conclusions: Mouth rinses can cause a negative effect on composite resins that increase in relation to time of contact.

Key words: Composite resins, mouth rinses, surface properties, loss of mass.

alimentos, líquidos como enjuague bucal, dentro de la masa de las resinas compuestas, produciendo una degradación. ${ }^{7}$ Dicha degradación está relacionada con el tipo de carga inorgánica, de acuerdo al tamaño y volumen, composición de la matriz y el grado de conversión del material resinoso. ${ }^{8,10} \mathrm{De}$ esta manera, se produce la difusión de agua dentro de la matriz resinosa ${ }^{6}$ provocando la ruptura de la unión entre el relleno inorgánico y la matriz, donde el agua y otros disolventes entran en el polímero conduciendo a la liberación de los productos, sobre todo en los oligómeros y monómeros. Lo que conduce a la pérdida de peso ${ }^{9} \mathrm{y}$ aumento de la rugosidad de la superficie externa del material. Debido a una degradación química de la matriz del polímero, dejando a las partículas de relleno parcialmente desprotegidas, ${ }^{11,12}$ fenómeno que ocurre de forma más evidente en procesos donde una foto polimerización incompleta se encuentra involucrada, ${ }^{6}$ apreciándose clínicamente aumento de micro filtración y disminución a la resistencia al desgaste. ${ }^{12}$

Los enjuagues bucales son sustancias que presentan en su formulación agua, agentes antimicrobianos, sales, conservantes, alcohol y peróxido de hidrógeno y constituyen una herramienta en la prevención, control de caries y enfermedades periodontales. ${ }^{6}$

Así también, en los diferentes programas preventivos de salud oral incorporan la utilización de colutorios en niños y adolescentes. Es importante 


\begin{tabular}{|c|c|c|c|}
\hline Material & \multicolumn{1}{|c|}{ Composición } & $\begin{array}{c}\text { Tiempo de } \\
\text { fotopolimerización }\end{array}$ & Fabricante/lote \\
\hline Grandio & $\begin{array}{l}\text { BisGMA, UDMA, TEGDMA. Rellenos: 87\% } \\
\text { en peso de bario, aluminio y silicato de boro } \\
\text { (1 micra), relleno de vidrio, nanorelleno } \\
\text { (SiO2) y 20 nm a 50 nm de cerámica. }\end{array}$ & 20 seg & (Voco, Germani), 1315343 \\
\hline Filtek Z250 XT & $\begin{array}{l}\text { BisGMA, UDMA, BisEMA, PEGMA y TEG- } \\
\text { MA, consta de zirconia / sílice de superfi- } \\
\text { cie modificada con una mediana de tamaño } \\
\text { de aproximadamente 3 micrones o menos, } \\
\text { partícula de sílice modificada de 20 nanómet- } \\
\text { ros no aglomerados/no agregados, la carga de } \\
\text { relleno es del 82\% por peso (68\% por volumen). }\end{array}$ & 20 seg & (3M Espe), N814073 \\
\hline
\end{tabular}

Tabla 1. Características de los materiales de restauración empleados.

conocer el efecto de estas sustancias en la estructura dental como también en los diferentes materiales utilizados en la cavidad bucal para indicar el momento idóneo para ser utilizado sin ninguna complicación tomando en cuenta su composición indicaciones y criterios de selección, evitando consecuencias posteriores a su uso.,

Los colutorios con alcohol presente en su composición, sumado a su $\mathrm{pH}$ bajo por la presencia de peróxido de hidrogeno, ${ }^{11,27}$ aumenta la sorción y so- lubilidad y por ende la degradación de la superficie de las resinas compuestas ${ }^{12,17,18}$ desencadenando el ablandamiento del material. Las mejoras en las propiedades mecánicas de los materiales resinosos suponen una mayor resistencia a este fenómeno, sin embargo no se encuentran muchos estudios al respecto.

Por esta razón se pretende mediante este estudio evaluar las variaciones de peso y rugosidad que sufren las resinas compuestas nano híbridas en con-

\begin{tabular}{|c|l|c|}
\hline Soluciones & \multicolumn{1}{|c|}{ Composición } & Fabricante/lote \\
\hline Listerine zero & $\begin{array}{l}\text { Agua, timol, eucaliptol, salicilato de metilo, men- } \\
\text { thol, propilen glicol, sorbitol, lauril sulfato de so- } \\
\text { dio, poloxámero 407, sacarina sódica 0,0606\%, su- } \\
\text { cralosa, benzoato de sodio, ácido benzoico, aroma } \\
\text { (d-Limonene). }\end{array}$ & Johnson \& Johnson 2796COC \\
\hline Listerine Cool mint & $\begin{array}{l}\text { Agua, sorbitol, alcohol, alcohol propílico, polox- } \\
\text { ámero 407, ácido benzoico, sacarina sódica, eu- } \\
\text { caliptol, sabor / aroma, timol, salicilato de metilo, } \\
\text { benzoato de sodio, mentol. }\end{array}$ & Johnson \& Johnson 0393COM \\
\hline Listerine Whitening & $\begin{array}{l}\text { Mentol, eucaliptol, alcohol etílico 8\%, fosfato de } \\
\text { sodio, lauryl sulfato de sodio, sacarina sódica, } \\
\text { agua, peróxido de hidrogeno, citrato de sodio, } \\
\text { sorbitol, aceite de castor hidrogenado, sucralo- } \\
\text { sa, ácido fosfórico, fosfato disódico. }\end{array}$ & Johnson \& Johnson 2752LZ \\
\hline
\end{tabular}

Tabla 2. Características de las soluciones utilizadas. 
tacto directo con los enjuagues bucales por diferentes períodos de tiempo, mediante balanza de precisión y rugosímetro digital.

\section{Materiales y método}

El estudio realizado fue experimental en vitro y no presentó conflictos de interés entre los integrantes y su aprobación está dada por el área de titulación de la facultad de Odontología de la Universidad Central del Ecuador. Se plantea un estudio de tipo experimental donde se realizaron 88 discos de resinas nanohíbridas, ambas de color A2, Grandio (VOCO) y Filtek Z250 XT (3M ESPE), (Tabla 1), fueron sometidos al contacto con tres diferentes enjuagues bucales, Listerine Zero, Listerine Coolmint y Listerine Whitening, por ser los comúnmente empleados en el mercado nacional ecuatoriano, previa verificación de contar con similares fechas de caducidad y de elaboración (Tabla 2), especificaciones contenidas en cada cuadro.

Los cuerpos de prueba fueron elaboradas por medio de una matriz de duralón, por la Empresa PROIMEC, de $5 \mathrm{~mm}$ de diámetro por $2 \mathrm{~mm}$ de espesor, se pulieron con lija de agua de granulación 360 marca Fandelli y se eliminaron irregularidades y se desinfectó con clorhexidina al 2\%. La matriz se colocó en una base de vidrio oscuro donde se obtuvo una superficie lisa de los cuerpos. Se aplicó directamente por capas las resinas empleadas tomando en cuenta las indicaciones del fabricante (Fig. 1), se colocó con un gutaperchero de titanio y se fotopolimerizó con una lámpara de luz LED marca (Woodpecker B) a una distancia de $1 \mathrm{~mm}$, la intensidad de la lámpara fotopolimerizadora fue evaluada antes del proceso y después de cada 10 cuerpos realizados mediante radiómetro (Demetron LED Radiometer, Kerr Sybron Dental Apecialities, Middleton, WI, USA).

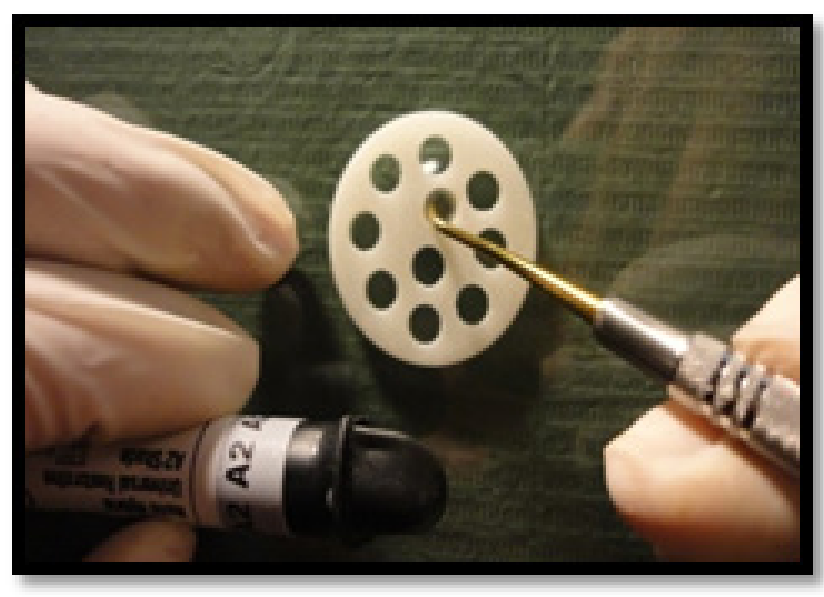

Figura 1. Colocación de la resina por capas de acuerdo a las indicaciones del fabricante.

Los cuerpos se dejaron en reposo por 60 minutos, luego fueron retirados de la matriz, y se procedió con el pulido mediante los discos sof-lex (3M ESPE) de grano grueso, fino, ultrafino en las dos superficies obteniendo cuerpos uniformes y libres de irregularidades (Fig. 2), posteriormente se colocó en cajas estériles individuales secas para mantenerlos libres de microorganismos. Las muestras obtenidas, se seleccionaron aquellos que no presentaron irregularidades, en ese momento fueron colocados en saliva artificial (Fig. 3), utilizando el salivsol compuesto por cloruro de sodio $0,084 \mathrm{~g}$, cloruro de potasio $0,120 \mathrm{~g}$, cloruro de calcio, dihidrato $0,015 \mathrm{~g}$, cloruro de

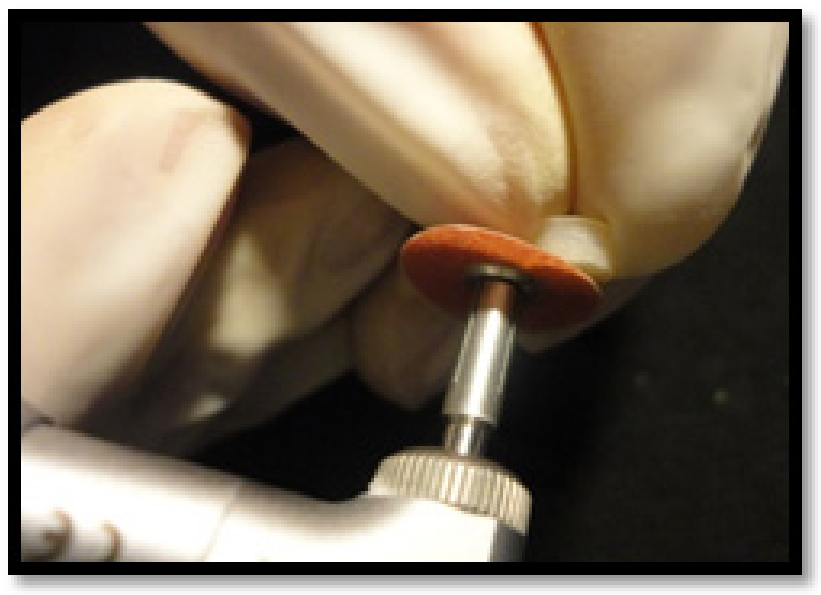

Figura 2. Pulido con discos sof-lex $3 M$ ESPE. 


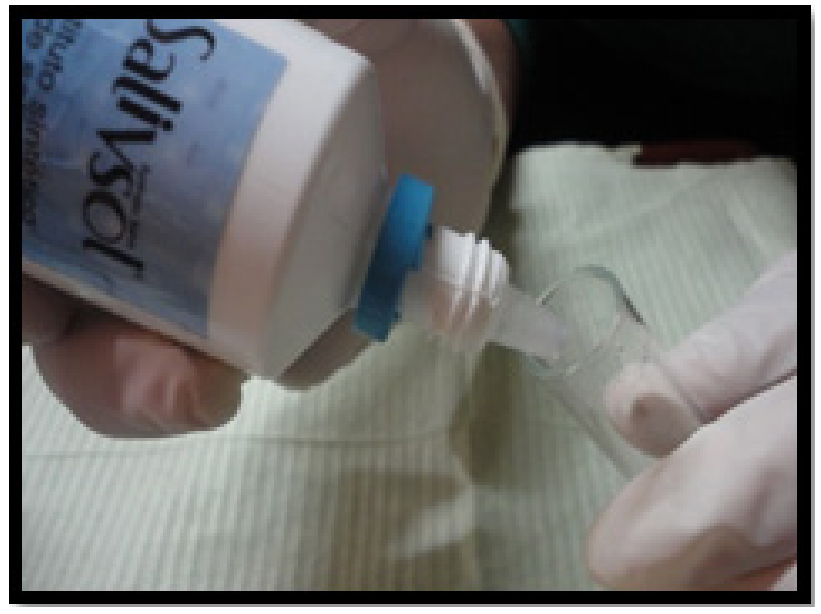

Figura 3. Las muestras colocadas en saliva artificial.

magnesio, hexahidrato 0,005g excipientes c.s.p. $100.000 \mathrm{ml}$, elaborado por laboratorios farmacéuticos Lamosan, lote 1307076, evitando su deshidratación a una temperatura ambiente de $37^{\circ} \mathrm{C}$, donde permanecieron por 12 horas realizándose a seguir el primer pesaje mediante la balanza de precisión GM 20 capacidad de $20 \mathrm{~g}$ x 0,001g e identificándolos cada uno (Fig. 4).

Los 88 cuerpos seleccionados, 44 muestras correspondieron a la resina Grandio (VOCO); fueron retirados dos muestras como grupo control y a su vez se las dividió en tres subgrupos conformados por (n: 14), las siguientes 44 muestras

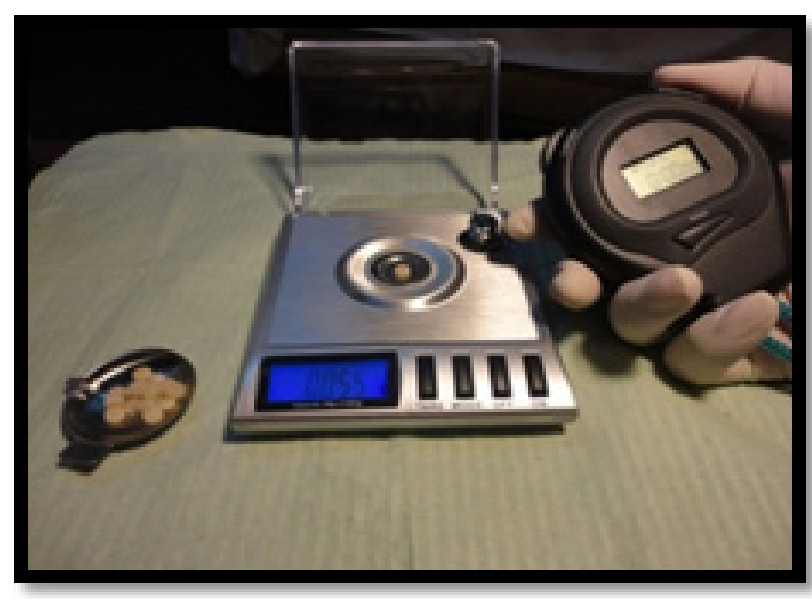

Figura 4. Pesaje inicial en Balanza de precisión digital.

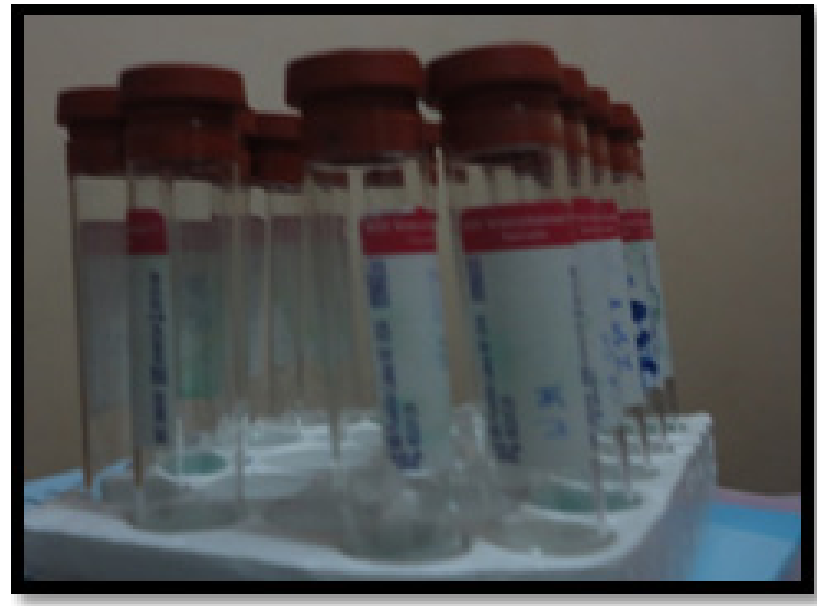

Figura 5. Los cuerpos de resina sumergidos en los enjuagues bucales empleados por 21 minutos.

que pertenecieron a la resina Filtek Z250 XT (3M ESP3), fueron divididas de igual forma.

De esta manera $20 \mathrm{ml}$ de cada enjuague fue dosificado en un envase apropiados y en el colocados los cuerpos de prueba según los subgrupos establecidos, donde fueron mantenidos durante 21 minutos consecutivos (Fig. 5), equivalentes a tres semanas de uso, considerando que el uso adecuado es de $20 \mathrm{ml}$ de enjuague bucal por 30 segundos dos veces al día, es decir 1 minuto diario indicaciones dadas por el fabricante, luego del tiempo expuesto se procedieron a retirarlos y se pesó cada uno de los cuerpos con la balanza de precisión, considerándose ésta la segunda medida de pesaje.

Tras el contacto con el enjuague bucal correspondiente, los cuerpos de prueba fueron removidos de la sustancia y colocados en una nueva saliva artificial durante 12 horas, para pasar luego a un nuevo contacto de enjuague bucal por 21 minutos realizándose otro pesaje, este procedimiento fue repetitivo hasta conseguir el contacto con el enjuague por 1092 minutos, correspondiente a los 3 años de uso. La eliminación de residuos de las substancias fue realizada siguiendo los cri- 


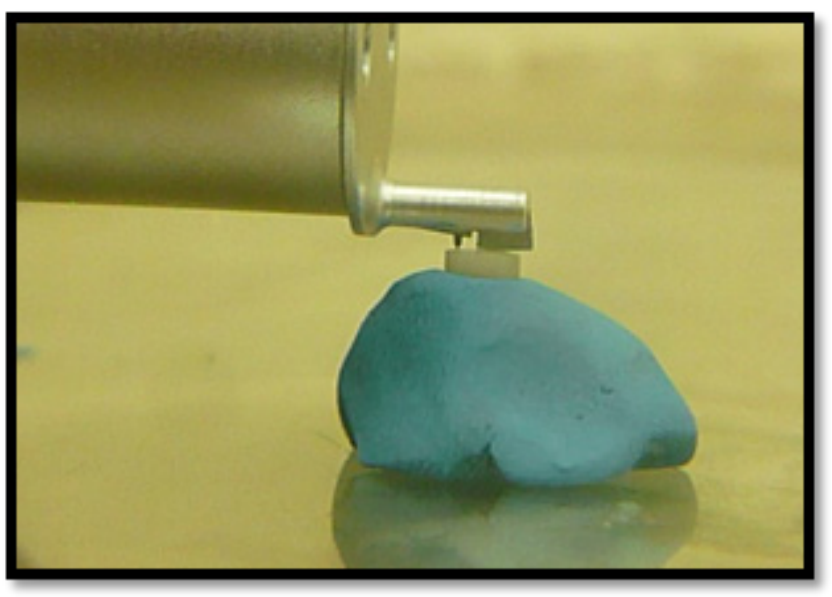

Figura 6. Evaluación en el Rugosímetro digital.

terios de bioseguridad y los cuerpos de prueba debidamente almacenados. Las pruebas de rugosimetría fueron realizadas en tres periodos de tiempo, inicial a los 546 minutos correspondientes a 18 meses de uso de colutorio bucal y a los 1092 minutos es decir a los 36 meses o 3 años de uso del mismo, empleando el rugosímetro digital (TAYLOR-HOBSON), Londres-Inglaterra con un alcance 0.3-100um.-10-300upulg, con una apreciación 0,003umm-0,08upulg, con un voltaje de 110V (Laboratorio de Metrología de la Escuela Politécnica del Ejército, Ecuador). (Fig. 6). La recolección de datos de los valores de pesaje en los distintos tiempos así como los resultados rugosidad fue colocado en tablas específicas y comparativas, adecuadamente diseñadas, que fueron sometidas a análisis estadísticos adecuados.

\section{Resultados}

Los resultados obtenidos se organizaron en una base de datos y se interpretaron mediante el programa SPSS (Statistical Package for the Social Sciences) software estadístico de la marca IBM 2010.

En el análisis de la rugosidad en los periodos de tiempo de 18 y 36 meses se observó que las mues- tras de resina aumentaron su rugosidad respecto al control, se observó que la resina Filtek Z250 XT (3M ESPE), presentó menor rugosidad al contacto con los diferentes enjuagues que los presentados por la Resina Grandio (VOCO), a priori no existen mayores variaciones ni con el tipo de enjuague ni con el tiempo (Gráfico 1 y 2).

Para la Resina Grandio (VOCO) se evidenciaron dos picos de interés, un primero obtenido en las resinas en contacto con Listerine CoolMint a los 18 meses y un segundo con Listerine Zero a los 36 meses (Gráfico 3), pero a su vez se obtuvieron los menores valores con Listerine Zero a los 18 meses y Listerine CoolMint a los 36 meses. Mediante la prueba ANOVA para los grupos en estudio determinó que si existen diferencias significativas en la rugosidad producida en las resinas Filtek Z250 XT (3M) y Grandio (VOCO), inmersas durante diferentes periodos de tiempos; 18 y 36 meses, en los diferentes enjuagues bucales $(p=0,001)$.

El test de Tukey, para establecer pares de estudio en forma comparativa, demostró, que existe aumento significativo de la rugosidad al exponer las resinas a las distintas marcas comerciales del enjuague bucal Listerine, observando la influencia del tiempo, donde al aumentar el tiempo de contacto simulado con el enjuague bucal la rugo-

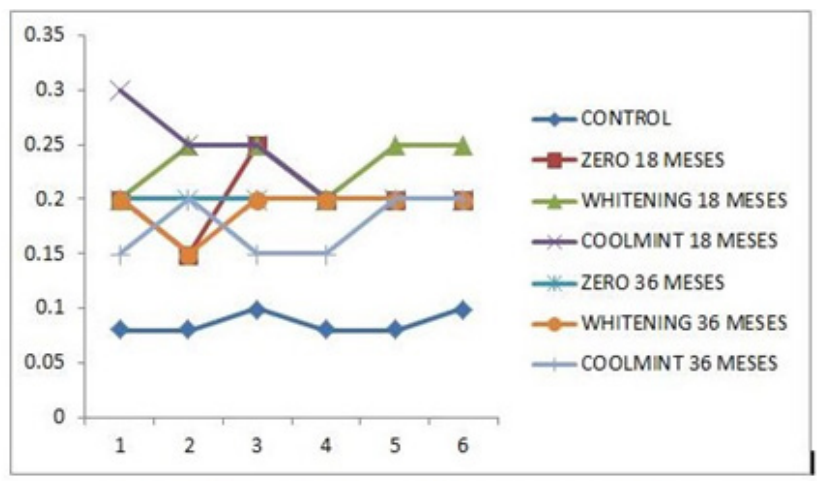

Gráfico 1. Rugosidad superficial Resina Filtek Z250 3M (um). 


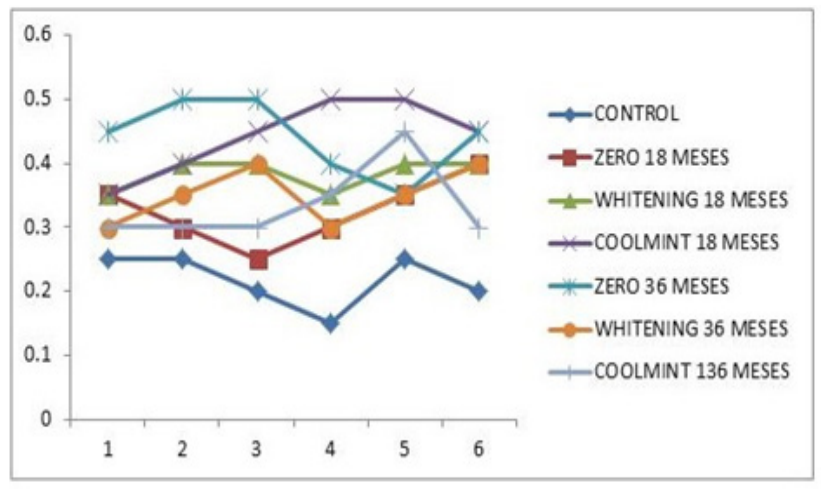

Gráfico 2. Rugosidad Superficial Resina Grandio Voco (um).

sidad aumento, comportamiento que se mantuvo independiente de la resina y del tipo de enjuague evaluado (Tabla 3).

En el presente estudio se analizó también la pérdida de peso (unidad de gramos), simulando desgastes a diferentes periodos de tiempo, siendo representativos los controles a 9, 18, 27 y 36 meses (Gráfico 4). Los datos refirieron que el peso inicial y luego de 9, 18, 27 y 36 meses para los dos tipos de resinas y los tres tipos de enjuagues empleados, las pérdidas de peso son mínimas, inclu- so en ciertas medidas se registró un valor mayor al inicial. Considerando la evaluación inicial del peso, y los diferentes tiempos de evaluación, los datos refirieron que el peso inicial considerado como línea base y aquel conseguido luego en los diferentes periodos de tiempo para los dos tipos de resinas y los tres tipos de enjuagues empleados, mostro una diferencia significativa $(\mathrm{p}<0,05)$ entre ellos, pero para los diferentes tiempos las variaciones de peso no son significativamente diferentes para los seis grupos analizados $(p>0,05)$, (Tabla 4).

\section{Discusión}

Actualmente la población, ha creado una mayor conciencia en su estado de su salud bucal, esto se traduce en la adopción de medidas higiénicas, el incremento en el uso de enjuagues bucales, sobre todo aquellos que presentan agentes blanqueadores, asociados a razones estéticas relacionadas al deseo de lucir dientes cada vez más blancos.

\begin{tabular}{|c|c|c|c|c|}
\hline RESINA & TIEMPO & LISTERINE & Media & Desv. típ. 2 \\
\hline \multirow[t]{7}{*}{ 3M ESPE } & & CONTROL & 0,0867 & 0,03 \\
\hline & \multirow[t]{3}{*}{18 MESES } & ZERO & 0,2000 & 0,035 \\
\hline & & WHITENING & 0,2333 & 0,033 \\
\hline & & COOLMINT & 0,2333 & 0,016 \\
\hline & \multirow[t]{3}{*}{36 MESES } & ZERO & 0,2000 & 0,054 \\
\hline & & WHITENING & 0,1917 & 0,032 \\
\hline & & COOLMINT & 0,1750 & 0,038 \\
\hline \multirow[t]{7}{*}{ VOCO } & & CONTROL & 0,2167 & 0,045 \\
\hline & \multirow[t]{3}{*}{ 18MESES } & ZERO & 0,3250 & 0,032 \\
\hline & & WHITENING & 0,3833 & 0,088 \\
\hline & & COOLMINT & 0,4417 & 0,041 \\
\hline & \multirow[t]{3}{*}{ 36MESES } & ZERO & 0,4417 & 0,038 \\
\hline & & WHITENING & 0,3500 & 0,061 \\
\hline & & COOLMINT & 0,3333 & 0,074 \\
\hline
\end{tabular}

Tabla 3. Rugosidad superficial media de los grupos. 


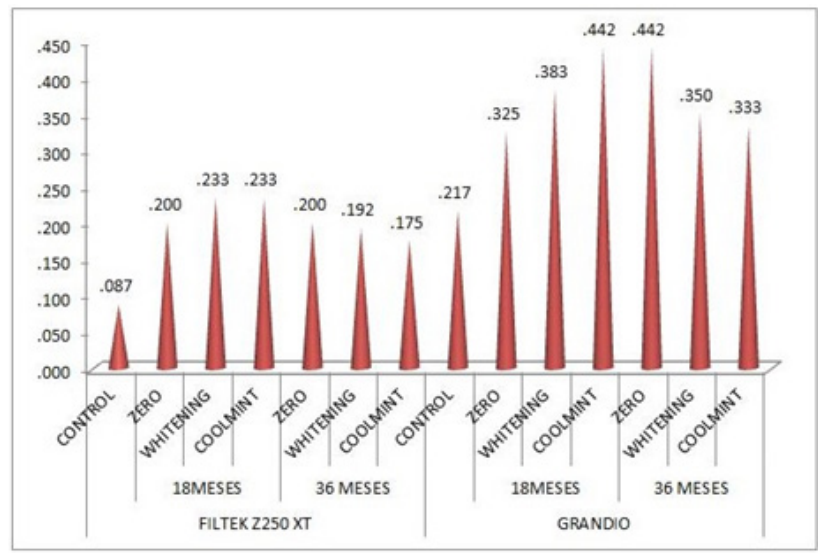

Gráfico 3. Rugosidad Superficial Media de los grupos de estudio (um).

En los adolescentes por la promoción de una salud adecuada y de dientes más estéticos, el uso de enjuagues bucales se va aumentando sin ninguna prescripción y por su facilidad de acceso; así también debido a los diferentes hábitos que van adquiriendo en su proceso de crecimiento adoptan medidas inadecuadas de higiene oral, incrementando la placa bacteriana por ende caries, alterando la estructura dentaria con el reemplazo de materiales odontológicos para que cumplan su función. ${ }^{8}$

En este proceso las resinas compuestas constituyen los materiales con más frecuencia utilizadas en Odontología debido a mejoras en su composición, procedimiento de unión, propiedades mecánicas, contracción de polimerización, absorción de líquido y de estabilidad en el medio ambiente oral. ${ }^{1-26}$ Sin embargo existen factores determinantes como enfermedades sistémicas que se pueden presentar en niños y adolescentes como la hemofila y cáncer, presentando en su sintomatología alteraciones a nivel bucal por los que es necesario el uso de enjuagues para prevenir la enfermedad periodontal en el desarrollo de la enfermedad base, de ahí la importancia de realizar estudios diferentes sobre la gran gama de antisépticos presentes que contribuyan como

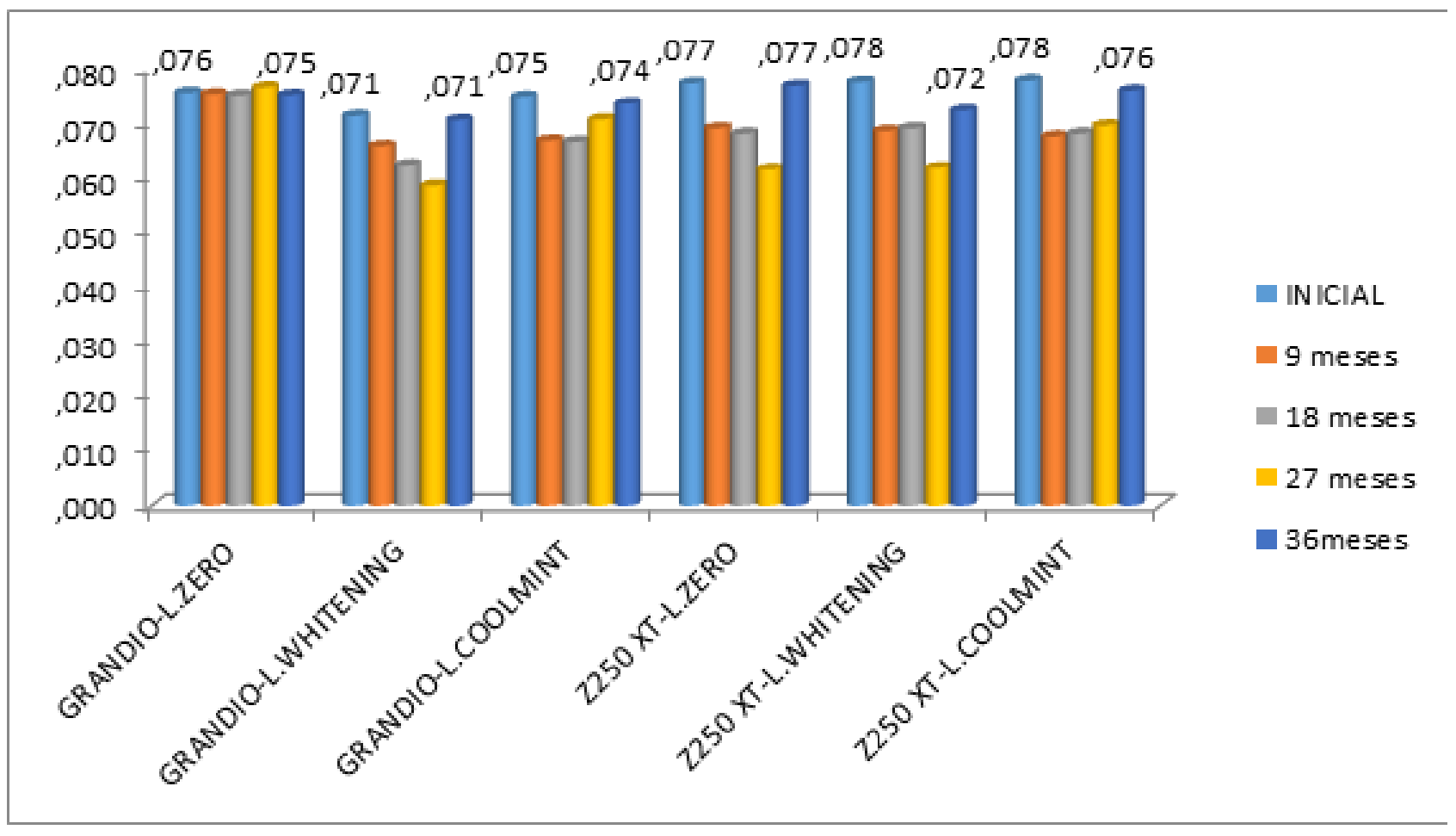

Gráfico 4. Pérdida de peso de los grupos de estudio. 


\begin{tabular}{|c|c|c|c|}
\hline & & $\begin{array}{c}\text { F Estadístico de la prueba } \\
\text { (Fisher Snedecor) de } \\
\text { varianzas. }\end{array}$ & Significancia. \\
\hline INICIAL & Inter-grupos & 6,741 & 0,001 \\
\hline 9 meses & Inter-grupos & 0,379 & 0,857 \\
\hline VAR00017 & Inter-grupos & 0,291 & 0,912 \\
\hline 18 meses & Inter-grupos & 0,449 & 0,808 \\
\hline 27 meses & Inter-grupos & 0,356 & 0,871 \\
\hline 36 meses & Inter-grupos & 1,205 & 0,346 \\
\hline
\end{tabular}

Tabla 4. Prueba ANOVA perdida de masa.

complemento de la higiene diaria, no produzcan alteraciones con los materiales de restauración utilizados en boca y sea biocompatible con la cavidad bucal. ${ }^{9}$

Sin embargo la degradación y envejecimiento de las restauraciones dentales asociada a la interacción con la composición de alimentos, saliva, productos de higiene bucal y bebidas en la cavidad oral, ${ }^{7}$ constituye un hecho innegable, de ahí el propósito de ejecutar este estudio.

Los resultados encontrados revelaron una pérdida de masa mínima de las muestras evaluadas, al comparar los grupos experimentales en relación al peso inicial y luego de 9, 18, 27 y 36 meses, fenómeno que ocurrió de forma similar para los dos tipos de resinas y los tres tipos de enjuagues bucales empleados. Sin embargo, la pérdida de masa se observó diferencias significativas en el peso inicial entre las resinas compuestas, al considerar los diferentes tiempos las pérdidas de masa no fueron significativamente diferentes entre los seis grupos analizados, coincidiendo con estudios similares ${ }^{23}$ observándose que la absorción de las sustancias por parte del material en los diferentes periodos de tiempo desarrolló variación en el peso inicial.
Los enjuagues bucales empleados en este estudio, contaron con diferente composición, sin embargo, no existieron diferencias fundamentales estadísticas entre su comportamiento, lo que puede estar explicado a otros componentes presentes en los enjuagues y no únicamente a la presencia o ausencia de alcohol. Los cambios fundamentales se dieron al considerar los diferentes periodos de tiempo de contacto, con resultados similares a estudios previos ${ }^{8}$ cuyos resultados demostraron aumento de rugosidad en todas las muestras, concluyendo que los enjuagues bucales y sobre todo aquellos que contenían peróxido de hidrogeno desencadenan un efecto mayor en la rugosidad de forma más evidente en materiales con base ionomérica. ${ }^{17,19}$

La elección de los enjuagues bucales empleados en el estudio fue realizada por las diferentes composiciones y en relación al consumo masivo por parte de usuarios que utilizan enjuagues bucales en el mercado ecuatoriano. Al considerar la rugosidad de la superficie de la resina compuesta a los 18 y 36 meses se observaron diferencias significativas entre los grupos, lo que concuerda con los estudios previos empleando peróxido de carbamida al $10 \%$ en contacto con resinas condensables donde se evidencio un aumento en la rugosidad superficial del material resinoso, ${ }^{24}$ 
coincidiendo con que el tipo de contacto de la muestra con el enjuague bucal utilizado afecta a la rugosidad de la superficie de la resina compuesta de forma más evidente cuanto este contacto se produce conjuntamente con el cepillado dental y en periodos de contacto prolongados. ${ }^{12}$

La evaluación de los cambios en la rugosidad de la superficie de un cemento de resina compuesta después de su almacenamiento en agua por diferentes periodos de tiempo, reveló un aumento significativo en la rugosidad de su superficie, ${ }^{21}$ que coincide con la presente investigación donde se observó la influencia del tiempo, al verificarse el aumentado en la rugosidad en los dos tipos de resina compuesta estudiadas al aumentar el tiempo de contacto independiente del enjuague bucal empleado.

Al analizar el efecto de tres bebidas (Wiskhy, Coca-Cola y Nimboz) sobre la rugosidad de la superficie en dos tipos de resinas, en contacto por diferentes intervalos de tiempo, se observó un aumento de la rugosidad en las superficie. ${ }^{23}$ Estudios similares, considerando la rugosidad de cuatro resinas compuestas fluidas expuestas a ácido y diferentes bebidas alcohólicas, constataron aumento de rugosidad en cada uno de los materiales probados, rugosidad superficial que se incrementaba con respecto a sus controles. ${ }^{23}$

En este estudio, en las muestras de resina Z250 se observó aumento de su rugosidad respecto al control, sin embargo, mayor variabilidad fue observada con muestras de la resina Grandio tras el contacto con Listerine CoolMint durante 18 meses. Estos resultados llevan a pensar en la necesidad de aumentar el número de cuerpos de prueba de cada grupo, buscando reducir esta variabilidad, pese a este inconveniente, fue evidente también que la menor variabilidad fue presentada cuando las muestras se mantuvieron en contacto con Listerine Zero por 18 meses, que al no contener alcohol en su composición aparentemente afecta de forma menos evidente la estructura resinosa.

Los dos materiales resinosos seleccionados, Z250 XT (3M ESPE) y Grandio (VOCO), fueron escogidos tomando como base el hecho de ser resinas empleadas con frecuencia en el medio odontológico ecuatoriano con composición y propiedades similares según especificaciones de la casa comercial, que en nuestro estudio mostraron un comportamiento similar, sin embargo con una evidente mayor estabilidad con la resina Z250 XT entre los periodos y con el contacto con las sustancias probadas. Mayores estudios requieren ser ejecutados con variaciones en cuanto a marcas de resinas compuestas considerando que el mercado odontológico abre una infinidad de posibilidades de materiales resinosos y día a día la industria de la prevención presenta diferentes enjuagues bucales cada vez más al alcance de la población.

\section{Conclusiones}

En las condiciones que este estudio fue ejecutado nos es factible concluir que, durante los diferentes tiempos de inmersión evaluados de los enjuagues bucales provocaron sobre las resinas compuestas pérdida de masa sin significación estadística dado que $p>0,05$. Las resinas compuestas en contacto con los diferentes enjuagues bucales comparados con los grupos de control y en tiempos de 18 y 36 meses presentaron aumento de su rugosidad siendo estos más evidentes en la resina Grandio. 


\section{Referencias bibliográficas}

1. Alkaline T, Genk G, Ceyhan Y, Bozkur F. The effect of mouthrinses on the color stability of sonic fill and a Nano hybrid composite. J Istanbul Univ. Fac Dent. 2016; 50: 17-23.

2. Ferracane J. Resin composite-State of the art. Dent Mater J. 2010; 27: 29-38

3. Velo M, Coelho L, Basting R, Aamaral F, Franca F. Longevity of restorations in direct composite resin: Literature review. Rev Gaúch Odontol. 2016; 64: 320-326.

4. Rashid B, Deepak. S, Ramakrishna. S, Navid S, Shetti S, Godhane A. Mouthrinses affect color stability of composite. J Conserv Dent. 2016: 355-9.

5. Ajaj R, Yousef M, Naga A. Effect of different curing modes on the degree of conversion and the microhardness of different composite restorations. Dent Hypotheses. 2015; 6: 109-16.

6. Azebedo M, Santos, B, Bovi. A, Baggio. A, Nunes. L, Lovadino R. Effect of curing light distance and different mouthinses on the Sorption and solubility of a nanofilled composite. Eur J of Esthet Dent. 2013; 8: 88- 102.

7. Clerehugh, V. TUGNAIT, A. Diagnosis and management of periodontal diseases in children and adolescents. J Periodontol. 2000; 26: 146-16.

8. Costa, F. Fernandes. L. Moura, E. Aguiar, Y. Santos. F. Cavalcanti, A. Oral health habits, prevalence of dental caries and dental erosion in adolescents. Rev Gaúch Odontol. 2017; 65: 202-7.

9. Barbosa, Bernal, L. Gallego, C. Sierra M. Comparación de los efectos de tres enjuagues en el manejo de la mucositis oral secundaria al tratamiento de leucemia linfoblástica aguda en niños. Rev Fac Odontol Univ Antioq. 2009; 20.

10. Razooki A, Aubi I. Solubility of nanofilled versus conventional composites. Pak Oral Dental J. $2014 ; 34: 1$.

11. Azebedo M, Santos B, Bovi. A, Baggio. A, Nunes L, Lovadino R. Effects of mouthwashes on knoop hardness and surface roughness of dental composites after different immersion times. Braz. Oral Res.

12. Sano T, Terossi G, Milori S, Colucci V, Milori C, Benitez C. The influence of mouthrinses and simulated tooth brushing on the surface roughness of a nanofilled composite resin. Braz Oral Res. 2012; 26: 209-14.

13. Falkensammer F, Vincent A, Wildburger A, Freudenthaler J. Color stability of different composite resin materials. J Prosthetic Dent. 2013: 378-83.

14. Sánchez S. ¿Son las restauraciones de resinas compuestas inertes y seguras? Revista ADM. 2010; 48(1):25-29.

15. Yildirim, A. Gulfem, E., Ferhan, E. Hisam, D. In vitro cytotoxity of indirect composite resins: Effect of storing in artificial saliva. Indian J Dent Res. 2010; 24: 81-86.

16. Jyothi K, Crasta S, Venugopal P. Effect of five commercial mouthrinses on the microhardness of a nanofilled resin composite restorative material: An in vitro study. J Conserv Dent.

17. Almeida G, Poskus L, Guimarães J, Silva E. The Effect of Mouthrinses on Salivary Sorption, Solubility and Surface Degradation of a Nanofilled and a Hybrid Resin Composite. Oper Dent . 2010; 35: 105-11.

18. Pallo, Chavez, Armas, Evaluación del grado de degradación de materiales restauradores en contacto con enjuagues bucales. Revista Científica Odontológica. 2010; 2: 14-16.

19. Sadaghiani L, Wilson M. Wilson N. Effect of five selected mouthrinse in surface roughness of the resin modified glass ionomer restorative materials. Dent Mater J. 2007; 23: 325-34.

20. Basting R. Fernandez, Bovi G, Campos I. Effects of a 10\% Carbamide peroxide bleaching agent on roughness and microhardness of packable composite resins. J Esthet Restor Dent. 2005; 17: 256-23.

21. Milleding P, Ahlgren F, Wennerberg A, Ortengren U, Karrison S. Effects roughness of the surface of composite resin cement water storage at different periods of time. Int J Prosthodont. 2005; 22: 34-9. 
22. Bansal K, Shashi R, Vidya S. Effect of alcoholic and non-alcoholic beverages on color stability and surface roughness of resin composite: A vitro study. J Conserv Dent. 2012; 15: 168-73.

23. Poggio C, Dagna A, Chiesa M, Colombo M, Scribanter A. Surface roughness of flowable resin composite eroded by acidic and alcoholic drinks. J Conserv Dent. 2012; 15: 34-38.

24. Evangelia C, Vouvoudi C, Theresia I, Baxevani G, Irini D. Dental dimethacrylate-based nanohybrid composite Kalore GC:

Kinetic study of its light-curing. J Taibah Univ Sci. 2016; 11: 63-71

25. Jarquín D, Bonilla, S. Aumento de la temperatura en la superficie dental durante la foto-polimerización. Odont. Vital. 2016; 25: $17-22$.

26. Lamas C, Lara G, De la Vega A. Clareamiento dental y restauraciones con resina compuesta - reporte de caso. KIRU. 2016; $13: 171-5$

27. Listerine, [Internet]: Listerine Professional. [Citado 5 abril 2015]. Disponible en: https://www.listerine.es/productos-listerine/ cuidado-total

Recibido: 09/01/2018

Aceptado: 15/03/2018

Correspondencia:

Paola Elisabeth Casanova Obando.paonova308@gmail.com 